\title{
Total Dorsal Pancreatectomy, an Alternative to Total Pancreatectomy: Report of a New Case and Literature Review
}

\author{
Simone Conci Andrea Ruzzenente Francesca Bertuzzo \\ Tommaso Campagnaro Alfredo Guglielmi Calogerolacono \\ Department of Surgery, Division of General and Hepato-pancreato-biliary, University of Verona Medical School, \\ Verona, Italy
}

\section{Keywords}

Parenchyma sparing pancreatectomy · Dorsal

pancreatectomy · Total dorsal pancreatectomy ·

Conservative pancreatectomy · Pancreatic surgery

\begin{abstract}
Background: Total dorsal pancreatectomy (TDP) is a conservative pancreatic resection that should be considered in cases of benign or low malignant tumors confined to the dorsal pancreas to preserve the viability of both digestive and biliary tracts, and to avoid the endocrine and metabolic consequences of total pancreatectomy. We report a new case of TDP and provide a literature review of this procedure. Methods: The case reported was a 35-year-old female patient with a solid pseudopapillary tumor. We resected the dorsal segment of the pancreas while preserving the common bile duct, gastroduodenal artery, and pancreaticoduodenal arcades, and the spleen and splenic vessels. The MEDLINE ${ }^{\circledR}$ and Embase ${ }^{\circledR}$ databases were searched for English language studies, case series, or case reports published through Au-
\end{abstract}

gust 31, 2017. Results: The postoperative course was uneventful and patient was discharged on postoperative day 11. The patient was alive and in good condition at the 10year follow-up. To date in English literature, there are only 3 reported cases of TDP, and all cases were patients with intraductal papillary mucinous neoplasia and pancreas divisum. There was no postoperative mortality, and 2 grade B pancreatic fistulas healed 1 month postoperatively. Conclusions: TDP is a feasible and safe operation for benign or low grade malignant pancreatic tumors involving the dorsal pancreas, as an alternative to total pancreatectomy.

(c) 2018 S. Karger AG, Basel

\section{Introduction}

The toolkit of modern pancreatic surgeon should include conservative techniques, such as duodenum-sparing pancreatic head resection [1], spleen-preserving distal pancreatectomy [2], central pancreatectomy [3-8], resection of ventral pancreas or uncinate process $[9,10]$, mid-

\section{KARGER}

(c) 2018 S. Karger AG, Basel

E-Mail karger@karger.com

www.karger.com/dsu
Calogero Iacono, MD

Department of Surgery, Unit of Hepato-Biliary-Pancreatic Surgery

University of Verona Medica School, “GB Rossi” University Hospital

P.le L.A. Scuro, 10, IT-37134 Verona (Italy)

E-Mail Calogero.Iacono@univr.it 

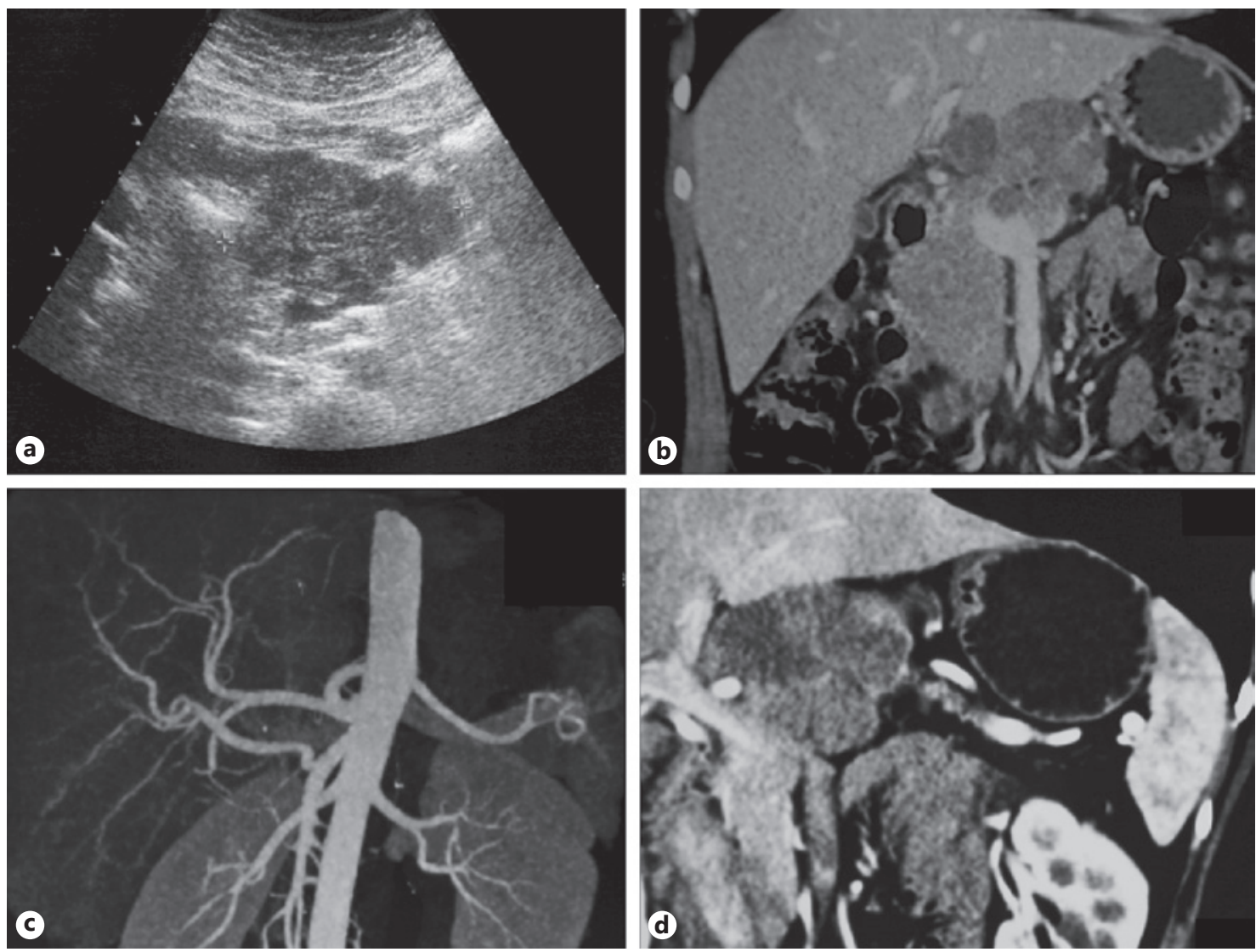

Fig. 1. Pre-operative ultrasonographic and computed tomographic scans of a 35-year-old female with persistent abdominal pain (case 1): (a) sonogram showing an $8 \mathrm{~cm}$ pancreatic mass; (b, c, and d) computed tomographic images confirming the presence of a pancreatic mass without signs of vascular infiltration and with hypertrophy of the ventral portion and uncinate process, atrophy of the body to tail (b and $\mathbf{d}$ ), and which was responsible for displacing the pancreas to the right of the gastroduodenal artery (c). dle-preserving pancreatectomy [11], total dorsal pancreatectomy (TDP) [12-14], and head dorsal pancreatectomy [15], as well as traditional pancreatic resection. In cases of benign or low grade malignant tumors confined to the dorsal pancreas, TDP should be considered to preserve the viability of both digestive and biliary tracts, and to avoid the endocrine and metabolic consequences of total pancreatectomy. The aims of this manuscript were to report a new case of TDP and to provide a literature review of this procedure.

Approval for TDP and this study was provided by our institutional ethics committee, and each patient or the patient's family gave informed consent for the procedure, and to publish the details of their case. The MEDLINE ${ }^{\circledR}$ and Embase ${ }^{\circledR}$ databases were searched for English language studies, case series, or case reports published through August 31, 2017. Search terms were "dorsal pancreatectomy", "total dorsal pancreatectomy", "segmental pancreatic resection", "conservative pancreatic surgery", and "ventral-sparing pancreatectomy". The reference lists of articles identified were manually searched to locate other articles of relevance.

\section{Case Report}

A 35-year-old female patient with persistent abdominal pain was referred to our hospital. Ultrasonography revealed an $8 \mathrm{~cm}$ pancreatic mass involving the dorsal head and neck of the pancreas (Fig. 1a). Computed tomography scan and abdominal magnetic resonance imaging (MRI) confirmed the presence of a pancreatic mass responsible for displacement to the right of the gastroduodenal artery (GDA) without signs of vascular infiltration and with atrophy of the body and tail of the pancreas and hypertrophy of the ventral portion uncinate process. Imaging showed characteristics suspicious for pancreatic solid pseudopapillary tumor. No signs of parenchymal infiltration or abdominal adenopathy indicating malignancy were observed (Fig. 1,2). Pre-opera- 

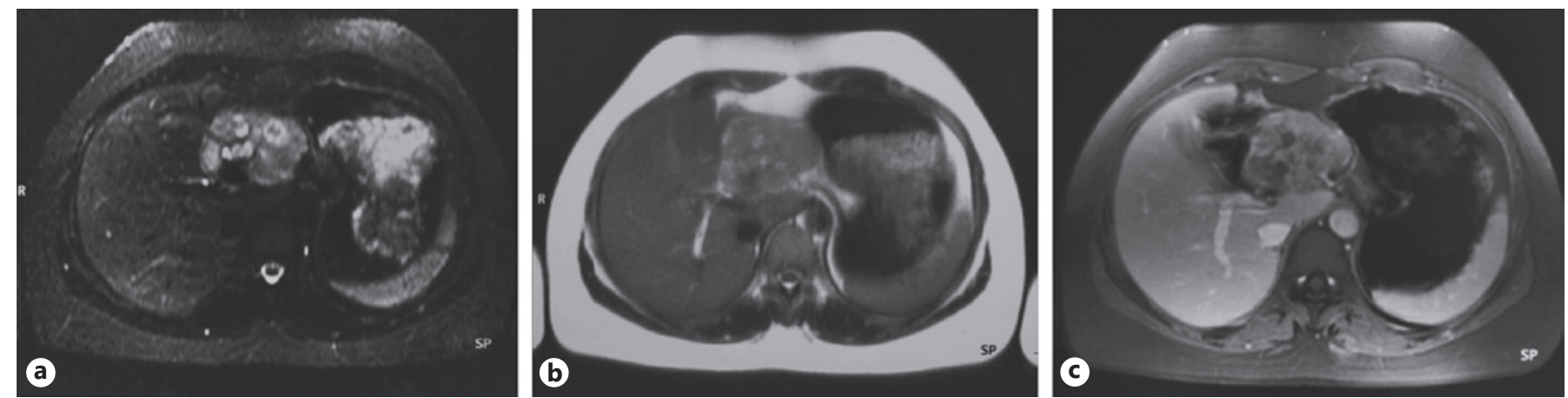

Fig. 2. Magnetic resonance images showing characteristics suspicious for pancreatic solid pseudopapillary tumor with signs of intratumoral hemorrhage. a T2-weighted fat saturation, (b) T2-weighted, and (c) contrast-enhanced portal phase T1-weighted images.

tive blood samples including tumor markers (carcinoembryonic antigen; carbohydrate antigenic determinant 19-9 [Ca 19-9]) and endocrine and exocrine pancreatic function tests were in the reference normal range. Considering the anatomical location of the tumor, atrophy of the distal pancreas, and hypertrophy of the ventral segment, the patient was scheduled for TDP with spleen preservation. The patient was informed that involvement of the transection margin, presence of invasive malignant disease, and intraoperative technical drawbacks could necessitate total pancreatectomy.

After laparotomy, intra-operative ultrasound of the pancreas and liver revealed a hypoechoic multiloculated $8.5 \mathrm{~cm}$ lesion with cystic and solid components in the dorsal portion of the pancreatic head and neck with atrophy of the body and tail. No other nodules were present in the remnant pancreatic parenchyma, and the ventral pancreas was hypertrophic. No lesions were identified in the liver. Intraoperative fine needle aspiration demonstrated the presence of a solid pseudopapillary tumor. The patients were submitted to the planned TDP. The postoperative course was uneventful and the patient was discharged on postoperative day 11.

Pathological examination of the surgical specimen confirmed the diagnosis of solid pseudopapillary tumor with atrophy and fibrosis of the distal pancreatic parenchyma. At the routine 10-year follow-up, the patient was in good general condition with no signs of endocrine or exocrine insufficiency, and color Doppler ultrasonographic evaluation of the vasculature confirmed patency of the splenic artery (SA) and splenic vein.

\section{Details of the Surgical Technique}

The pancreatic region is reached by dissecting the gastroepiploic ligament, preserving the gastroepiploic vessels. Kocher's maneuver should be avoided or carefully performed in order to preserve the posterior pancreaticoduodenal arcades. To identify the intra-pancreatic tract of the common bile duct (CBD) in the ventral segment, a catheter can be inserted into the CBD to the duodenum through the cystic duct; catheterization can also be performed after choledochotomy. Alternatively, pre-operative endoscopic placement of biliary and/or pancreatic plastic stents can be performed to facilitate intra-operative identification of the CBD and the pancreatic duct. The common hepatic artery, GDA, anterior superior pancreaticoduodenal artery, and SA are identified and looped. The superior mesenteric vein is then dissected from the portal groove of the pancreatic neck. The pancreatic tail is mobilized while identifying and preserving the SA and splenic vein by ligating arterial and venous collateral branches.

Pancreatic resection is performed from left (tail) to right (head). At the head of the pancreas, the dorsal segment is dissected as described previously [15] stepwise from the duodenal wall toward the CBD plane while preserving the GDA and the anterior superior pancreaticoduodenal artery. Santorini's duct is identified, dissected, and ligated at its outlet into the duodenum followed by dissection of the pancreatic parenchyma along the anterior surface of the CBD.

To complete the TDP, the fusion plane between dorsal and ventral segments is identified and carefully dissected. The main pancreatic duct in the ventral segment of the dissected parenchymal surface is identified, dissected, and ligated. Branch ducts identified on the surface of the ventral segment are then sutured in an interrupted pattern (Fig. 3). Frozen sections of the pancreatic parenchymal margin should be collected and analyzed in all cases.

\section{Literature Review}

To date in the literature, there are only 3 reported cases of TDP [12-14], and all 3 patients were diagnosed with intraductal papillary mucinous neoplasia (IPMN) with pancreas divisum (Table 1). Two patients had extensive intraductal mucinous tumors with areas of ranging from severe dysplasia to carcinoma in situ $[12,13]$, and 1 patient had non-invasive IPMN with foci of severe dysplasia involving several branch ducts and parts of the cephalic duct [14]. The transection margin was free of tumor in all cases. There was no postoperative mortality, and 2 grade $B$ pancreatic fistulas healed 1 month after surgery $[12,14]$. All patients were alive at the last reported follow-up. The first patient was alive 12 months postoperatively with insulin-dependent type II diabetes without exocrine insufficiency [12]; the second patient was alive 6 years postoperatively with insulin-dependent type II diabetes, signs of mild exocrine insufficiency, and without recurrence [13]; and the third patient was alive 18 months postoperatively with insulin-dependent type II diabetes without exocrine insufficiency and free of recurrence on MRI [14] (Table 1). 


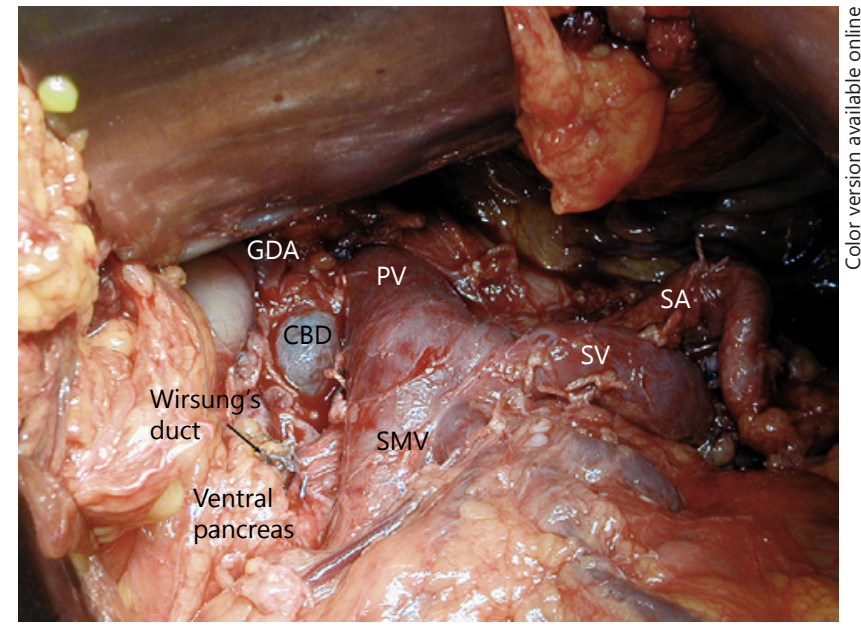

Fig. 3. Intraoperative field after total dorsal pancreatectomy with preservation of the ventral pancreas. GDA, gastroduodenal artery; $\mathrm{CBD}$, common bile duct; PV, portal vein; SMV, superior mesenteric vein; SV, splenic vein.

\section{Discussion}

Several authors recently reported successful conservative pancreatic resection of the dorsal segment, including the 3 cases of TDP [12-14], and 3 cases of partial dorsal pancreatectomy defined as ventral pancreas-preserving pancreatic head and body resection [16], anterior segmentectomy of the pancreatic head [17], and head dorsal pancreatectomy [15].

TDP is a conservative operation that allows for complete removal of the pancreatic mass while avoiding biliary and digestive tract resection/reconstruction and preserving endocrine and exocrine function. In selected cases, TDP represents the only alternative to total pancreatectomy and difficulties managing the subsequent "fragile" diabetes. Although postoperative diabetes was common in the reported cases of TDP, diabetes following TDP is easier to control than that resulting from total pancreatectomy because the glucose-stabilizing effects of glucagon may be maintained [12-14]. As reported in Table 1, all the patients submitted to TDP had a benign or low grade malignant tumor. In our opinion, a correct indication to TDP could be the presence of a benign or low malignant neoplasm, such as IPMN, pancreatic neuroendocrine tumors, and SPT confined in the dorsal segment of the pancreas without involvement of ventral segment or Wirsung's duct, as alternative of total pancreatectomy. In case of IPMN, if the entire main pancreatic duct is involved, ventral pancreas should not be preserved. In this case, duodenum preserving pancre- atic resection could be considered [1]. In case of invasive or malignant neoplasm suspicious intraoperatively or at the frozen section, TDP should be converted to total pancreatectomy with standard lymphadenectomy. Moreover, according to the postoperative results reported in literature we can assume that TDP could be safely proposed with acceptable postoperative morbidity. No postoperative mortality has been reported with a rate of clinically significant pancreatic fistula of 50\% (2/4) healed within one month by conservative management.

\section{Anatomical and Embryological Bases}

The pancreas is composed of 2 embryological segments, dorsal and ventral primordia. During the sixth week, the ventral primordium, together with the developing bile duct, rotates clockwise behind the duodenum and the dorsal primordium [18]. The differences in embryologic origins reflect histological characteristics. Compared with the dorsal pancreas, the ventral pancreas is characterized by the presence of smaller and more closely packed lobuli, irregular rather than uniform islets of Langerhans, and rich immunostaining with anti-pancreatic polypeptide [19]. Uchida et al. [19], Kimura and Nagai [20], and Sakamoto et al. [21] reported autoptical pancreatic anatomical studies on 20,40 , and 31 cases, respectively [19-21]. In each of these studies, the authors showed that the head of the pancreas can be removed while preserving vascular arcades and branches to the duodenum, the CBD, and the papilla of Vater [19], and that there is an anatomical fusion plane between the dorsal and ventral pancreas that contains small pancreatic ducts and vascular collateral branches $[20,21]$. These studies confirm the theoretical feasibility of segmental pancreatic resection along this fusion plane. Resections of both the dorsal and ventral pancreas have been reported, demonstrating their technical feasibility and safety $[9,10$, $21,22]$.

The presence of pancreas divisum, the lack of fusion between the dorsal and ventral pancreas during embryological development, is a favorable anatomical condition that can allow pancreatic segmental resection; however, the procedure is possible even in the absence of this condition if the correct morphology of the pancreatic fusion plane is present $[20,21]$.

\section{Technical Aspects}

The first to describe and publish the surgical techniques of TDP was a group from the Massachusetts General Hospital in 2002 in an asymptomatic 71-year-old fe- 
Table 1. Clinical and pathological characteristics of the patients submitted to total dorsal pancreatectomy reported in literature

\begin{tabular}{lllllllr}
\hline Authors & Year & Age & Gender & Histology & Pancreatic fistula & $\begin{array}{l}\text { Exocrine } \\
\text { insufficiency }\end{array}$ & $\begin{array}{c}\text { Diabetes } \\
\text { Survival, } \\
\text { months }\end{array}$ \\
\hline Thayer et al. [12] & 2002 & 71 & Female & IPMN & Grade B & - & + \\
Talbot et al. [13] & 2005 & 51 & Female & IPMN & - & + & + \\
Scatton et al. [14] & 2006 & 45 & Male & IPMN & Grade B & - & + alive \\
Current case & 2018 & 35 & Female & SPT & - & - & - \\
\hline
\end{tabular}

IPMN, intraductal papillary mucinous neoplasia; SPT, solid pseudopapillary tumor.

male with pancreas divisum, and an IPMN involving the dorsal segment [12]. However, according to the patient's clinical history, it seems that TDP was performed for the first time in 1998 by Imrie et al. [13] although the authors published the case in 2005.

The technique described by Thayer et al. [12] provides the advancing of a catheter after cholecystectomy through the cystic duct into the CBD through the papilla into the duodenum to better identify the CBD during parenchymal dissection and to verify its integrity at the end of the operation. The authors also ligated the GDA to expose the supra-pancreatic portal vein and to facilitate dissection of the pancreatic neck. Pancreatic resection began at the pancreatic neck to allow for inspection and assessment of intraductal involvement of the tumor. In patients with pancreas divisum, the fusion plane between dorsal and ventral segments at the pancreatic head was easily identified at the superior-posterior portion of the pancreas and was described as a fibrous septum that allowed relatively simple and bloodless dissection and separation of the pancreatic segments along a plane passing through the anterior surface of the $\mathrm{CBD}$, which was entirely preserved. The dorsal segment was dissected en-bloc with the minor papilla, and the body and tail of the pancreas were then removed while preserving the spleen, using Warshaw's technique [2]. On completion of the surgery, the first portion of the duodenum developed ischemic injury that necessitated resection of the antrum and proximal duodenum followed by gastrojejunostomy.

Even though we should praise the innovative idea that brings in the toolkit of a pancreatic surgeon a new conservative operation, some comments should be made on the technique performed. First, hypovascularization of the duodenum forced the authors to resect and reconstruct the digestive tract; therefore, preserving the GDA is recommended to avoid ischemic injury. Second, the decision to transect the pancreas at the neck with separate resection of the dorsal segment of the head and the body tail is controversial. There are no clear advantages of this technique compared with en-bloc resection as we and Sauvanet and colleagues [14] propose. Third, spleen preservation with ligation of the splenic vessels is a feasible and safe technique but it should be reserved for specific cases because splenic infarcts and abscesses have been reported $[2,23$, 24].

The case reported by Talbot et al. [13] suffered a long and troubled diagnostic process and was characterized by issues similar to the previous case with 2-step pancreatic dorsal segment resection after parenchymal transection at the neck and the need for digestive reconstruction (because of previous endoscopic cystic duodenal drainage). During laparotomy and after excision of the previous cystojejunostomy, differing firmness in the ventral and the dorsal pancreas suggested the authors to perform a TDP. Pancreatic resection began with parenchymal transection at the neck as described by Thayer et al. [12] and proceeded with resection of the dorsal segment along the embryological fusion plane between the ventral and the dorsal segments. The second portion of the duodenum and the retroduodenal portion of the CBD were then resected followed by resection of the pancreatic body and tail while preserving the spleen and its vessels. Digestive and biliary continuity was ensured by an end-to-end duodenum-jejunal anastomosis and an end-to-side bile ductjejunal anastomosis.

From a technical perspective, the procedure proposed by Scatton et al. [14] was performed more correctly. Kocher's maneuver was not performed and both the GDA and the posterior pancreaticoduodenal arcade were preserved to minimize the risk of duodenal ischemia. After mobilizing and taping the pancreatic neck, pancreatic transection began $2-3 \mathrm{~mm}$ from the duodenal wall along a plane passing through the 
anterior surface of the CBD. The dorsal pancreatic duct was divided and ligated $3 \mathrm{~mm}$ away from the duodenal lumen, and TDP was completed by resecting the pancreatic body and tail while preserving the spleen and its vessels.

In our patient, we performed pancreatic resection from left to right after complete mobilization of the pancreatic body and tail. As suggested by Scatton et al. [14] we carefully preserved the GDA and avoided Kocher's maneuver.

\section{Conclusions}

Our case and previously reported cases confirm that TDP is a feasible and safe operation for benign or low malignant pancreatic tumors involving the dorsal pancreas as an alternative to total pancreatectomy.

\section{Disclosure Statement}

The authors declare no conflicts of interest.

\section{References}

1 Beger HG, Krautzberger W, Bittner R, Buchler M, Limmer J: Duodenum-preserving resection of the head of the pancreas in patients with severe chronic pancreatitis. Surgery 1985;97:467-473.

2 Warshaw AL: Conservation of the spleen with distal pancreatectomy. Arch Surg 1988;123: 550-553.

3 Dagradi A, Serio G: Pancreatectomia Intermedia. Enciclopedia Medica Italiana, vol XI: Pancreas. Firenze, USES Edizioni Scientifiche, 1984, pp 850-851.

4 Iacono C, Bortolasi L, Serio G: Is there a place for central pancreatectomy in pancreatic surgery? J Gastrointest Surg 1998;2:509-516.

5 Iacono C, Bortolasi L, Serio G: Indications and technique of central pancreatectomy-early and late results. Langenbecks Arch Surg 2005;390:266-271.

6 Iacono C, Bortolasi L, Facci E, Nifosi F, Pachera S, Ruzzenente A, Guglielmi A: The Dagradi-Serio-Iacono operation central pancreatectomy. J Gastrointest Surg 2007;11:364-376.

7 Iacono C, Bortolasi L, Serio G (eds): Central Pancreatectomy. Disease of the Pancreas Current Surgical Therapy, ed. Springer-Verlag, 2008, pp 425-440.

8 Iacono C, Verlato G, Ruzzenente A, Campagnaro T, Bacchelli C, Valdegamberi A, Bortolasi L, Guglielmi A: Systematic review of central pancreatectomy and meta-analysis of central versus distal pancreatectomy. Br J Surg 2013;100:873-885.

9 Takada T: Ventral pancreatectomy: resection of ventral segment of the pancreas. J Hepatobiliary Pancreat Surg 1993;1:36-40.
10 Ryu M, Takayama W, Watanabe K, Honda I, Yamamoto H, Arai Y: Ventral pancreatic resection for adenoma and low-grade malignancies of the head of the pancreas. Surg Today 1996;26:476-481.

11 MiuraF,TakadaT,AmanoH,YoshidaM,ToyotaN, Wada K:Middle-segment-preservingpancreatectomy.JAm CollSurg 2007;204:720-722.

12 Thayer SP, Fernandez-del Castillo C, Balcom JH, Warshaw AL: Complete dorsal pancreatectomy with preservation of the ventral pancreas: a new surgical technique. Surgery 2002;131:577-580.

13 Talbot ML, Foulis AK, Imrie CW: Total dorsal pancreatectomy for intraductal papillary mucinous neoplasm in a patient with pancreas divisum. Pancreatology 2005;5(2-3):285288.

14 Scatton O, Sauvanet A, Cazals-Hatem D, Vullierme MP, Ruszniewski P, Belghiti J: Dorsal pancreatectomy: an embryology-based resection. J Gastrointest Surg 2006;10:434-438.

15 Iacono C, Ruzzenente A, Conci S, Xillo L, Guglielmi A: Head dorsal pancreatectomy: an alternative to the pancreaticoduodenectomy for not enucleable benign or low-grade malignant lesions. Pancreatology 2014;14:419-424.

16 Ohwada S, Ogawa T, Kasahara M, Kawate S, Koyama T, Izumi M, Takashi N, Morishita Y: Ventral pancreas-preserving pancreatic head and body resection. Hepatogastroenterology 2001;48:1622-1624.

17 Sakamoto Y, Tanaka N, Nagai M, Nobori M, Otani T, Makuuchi M: Anterior segmentectomy of the pancreatic head for islet cell tumors. Pancreas 2002;24:317-319.
18 Borghi F, Gattolin A, Garbossa D, Bogliotto F, Garavoglia M, Levi AC: Embryologic bases of extended radical resection in pancreatic cancer. Arch Surg 1998;133:297-301.

19 Uchida T, Takada T, Ammori BJ, Suda K, Takahashi T: Three-dimensional reconstruction of the ventral and dorsal pancreas: a new insight into anatomy and embryonic development. J Hepatobiliary Pancreat Surg 1999;6: 176-180.

20 Kimura W, Nagai H: Study of surgical anatomy for duodenum-preserving resection of the head of the pancreas. Ann Surg 1995;221: 359-363.

21 Sakamoto Y, Nagai M, Tanaka N, Nobori M, Tsukamoto T, Nokubi M, Suzuki Y, Makuuchi M: Anatomical segmentectomy of the head of the pancreas along the embryological fusion plane: a feasible procedure? Surgery 2000;128:822-831.

22 Sauvanet A, Gaujoux S, Blanc B, Couvelard A, Dokmak S, Vullierme MP, Ruszniewski P, Belghiti J, Levy P: Parenchyma-sparing pancreatectomy for presumed noninvasive intraductal papillary mucinous neoplasms of the pancreas. Ann Surg 2014;260:364371 .

23 Aldridge MC, Williamson RC: Distal pancreatectomy with and without splenectomy. $\mathrm{Br} \mathrm{J}$ Surg 1991;78:976-979.

24 Ferrone CR, Konstantinidis IT, Sahani DV, Wargo JA, Fernandez-del Castillo C, Warshaw AL: Twenty-three years of the Warshaw operation for distal pancreatectomy with preservation of the spleen. Ann Surg 2011; 253:1136-1139. 